\title{
Calibration of NAOS and CONICA static aberrations
}

\section{Experimental results}

\author{
M. Hartung ${ }^{1}$, A. Blanc ${ }^{2,3}$, T. Fusco ${ }^{2}$, F. Lacombe $^{4}$, L. M. Mugnier ${ }^{2}$, G. Rousset ${ }^{2}$, and R. Lenzen ${ }^{1}$ \\ 1 Max-Planck-Institut für Astronomie, Königstuhl 17, 69117 Heidelberg, Germany \\ e-mail: hartung@mpia.de \\ 2 Office National d'Études et de Recherches Aérospatiales, Département d'Optique Théorique et Appliquée, \\ BP 72, 92322 Châtillon Cedex, France \\ 3 Laboratoire des Signaux et Systèmes, École Supérieure d'Électricité, Plateau de Moulon, 91192 Gif-sur-Yvette, France \\ 4 Observatoire Paris-Meudon, LESIA, Place Jules Janssen, 92195 Meudon Cedex, France
}

Received 16 July 2002 / Accepted 25 October 2002

\begin{abstract}
NAOS is the first adaptive optics (AO) system of the Very Large Telescope and will provide CONICA with diffraction-limited images. CONICA is a near infrared camera that offers a variety of imaging and spectroscopic observing modes. A technique will be described to benefit of the AO system to correct not only for atmospheric turbulence but also for the internal optical aberrations of the high-resolution camera and the beam splitters of NAOS. The aberrant optical components in the light path of CONICA as well as the beam splitters are outside of the AO loop and therefore no self-acting correction is possible. Independently of the AO wavefront sensor, a separate measurement of these aberrations using a method called phase diversity allows one to predict for a certain instrument configuration the corresponding aberrations. They are quantified by sets of Zernike coefficients that are rendered to the adaptive optics. This technique turns out to be very flexible and results in a further improvement of the optical overall performance. The application of phase diversity to the instrument is investigated in a preceding paper (Blanc et al. 2003). In the present paper we present in detail the instrumental implementation of phase diversity, the obtained calibration results, and the achieved gain in optical performance.
\end{abstract}

Key words. instrumentation: adaptive optics

\section{Introduction}

NAOS-CONICA is the first adaptive optics (AO) system of the Very Large Telescope (VLT) and saw its first light in November 2001 (Brandner et al. 2002). The Nasmyth Adaptive Optics System NAOS (Rousset et al. 1998; Rousset et al. 2000) delivers diffraction-limited images to the Coudé ${ }^{1}$ Near Infrared CAmera CONICA (Lenzen et al. 1998; Hartung et al. 2000). To retrieve the maximum possible performance of the system in terms of Strehl ratio ${ }^{2}$ (SR) a method has been developed to calibrate the remaining degradation of the image quality induced by its optical components. Defaults of the wavefront attributed to any degradation within the AO loop (common path) are seen directly by the AO wavefront sensor (WFS) and thus the AO system can correct for these aberrations automatically.

\footnotetext{
Send offprint requests to: T. Fusco,

e-mail: thierry.fusco@onera.fr

1 The notation Coudé has historical origins as CONICA originally was planned for the Coudé focus.

2 The SR ratio is a common way to describe the quality of the point spread function. It is given by the ratio of the measured and the theoretical diffraction-limited peak intensity.
}

This is not the case for a degradation of image quality induced by components outside the AO loop. An experimental setup has been applied which allows one to sense the wavefront of the light which has passed the whole system without making use of the AO wavefront sensor. Therefore we draw on a well-known method called phase diversity (Gonsalves 1982; Paxman et al. 1992). It turns out that a number of theoretical and experimental constraints have to be examined before reliable results can be obtained in sensing the wavefront via phase diversity (PD). We focused on this in a precedent paper (Blanc et al. 2003), hereafter Paper I. In this second paper we first give a brief description of the instrument (Sect. 2). Then we focus on the experimental setup which enables us to calibrate the variety of beam splitters, filters and camera objectives. The design constraints for the implementation of PD are illustrated, and the resulting setup as well as the procedure to obtain the appropriate input data for PD are described (Sect. 3).

Because of the huge number of instrument modes it is not feasible to perform the PD calibration for each possible configuration. We explain how the wavefront degradations of the different optical components are disentangled. Then, the individual parts of the optical train can be calibrated separately and it 
is no longer required to do this for every possible combination. In detail, we will allocate the wavefront error to the dichroic mirrors of NAOS (beam splitter between wavefront sensor and imaging path), to the CONICA filters and camera objectives (Sect. 4).

Thereafter, the sensed wavefront errors are used to calculate the corresponding SRs. These are compared to the SRs directly determined from the images and the consistency is verified (Sect. 5). Finally, after presentation of the complete calibration procedure and its results, the measured wavefront errors are rendered in terms of Zernike coefficients to the AO system to demonstrate the gain in overall performance after closed loop correction (Sect. 6).

\section{The instrument}

This section is dedicated to a brief description of the instrument NAOS-CONICA. In particular, we emphasize the subdevices as much as the aspects of design which are important with respect to the static wavefront error estimation by phase diversity. Figure 1 gives an overview of the VLT instrument. NAOS is installed at one of the Nasmyth foci. It picks up an $\mathrm{f} / 15$ beam, corrects for atmospheric turbulence and hands on again an $\mathrm{f} / 15$ beam providing CONICA with diffraction-limited images. Having passed the Nasmyth focal plane, the beam is led to a first collimating parabola. Then it is reflected successively onto the tip-tilt (TTM) and the deformable mirror (DM). The following dichroic mirror separates the optical train into the imaging path and the wavefront sensing path. NAOS offers five different dichroic beam splitters to adapt for the flux and the spectral characteristics of the guide star. In the imaging path the light is refocused onto the entrance focal plane of CONICA, which is located behind the entrance window in the cold cryostat. Between NAOS and CONICA an atmospheric dispersion compensator (ADC) can be slid in in case of a high zenith angle.

The wavefront sensing path consists of a field selector (Spanoudakis et al. 2000) and two wavefront sensors. They are located between the dichroic mirror and the WFS input focus. For the sake of clarity these components are not shown in Fig. 1. The two wavefront sensors, one in the visible and one in the near infrared spectral range, enhance the sky coverage of possible guide stars. The field selector chooses the guide star in a 2 arcmin field of view and allows differential object tracking, pre-calibrated flexure compensation and counter-chopping. In combination with the deformable mirror it is also able to correct for a certain amount of defocus, as needed when the prisms of the atmospheric dispersion compensator are shifted into the beam. Note that this ability of focus correction offers a possibility to perform PD measurements that we will refer to later on (Sect. 3.2).

The high angular resolution camera CONICA is equipped with an Aladdin array $(1 \mathrm{~K} \times 1 \mathrm{~K})$ covering the $1-5 \mu \mathrm{m}$ spectral region. Splitting the wavelength region into two parts (1 to $2.5 \mu \mathrm{m}$ and 2.0 to $5 \mu \mathrm{m}$ ) allows us to keep the light path achromatic. Therefore the four different pixel scales are realized by seven cameras (Table 1). To each pixel scale a camera is associated with the short wavelengths region (S-camera) and another

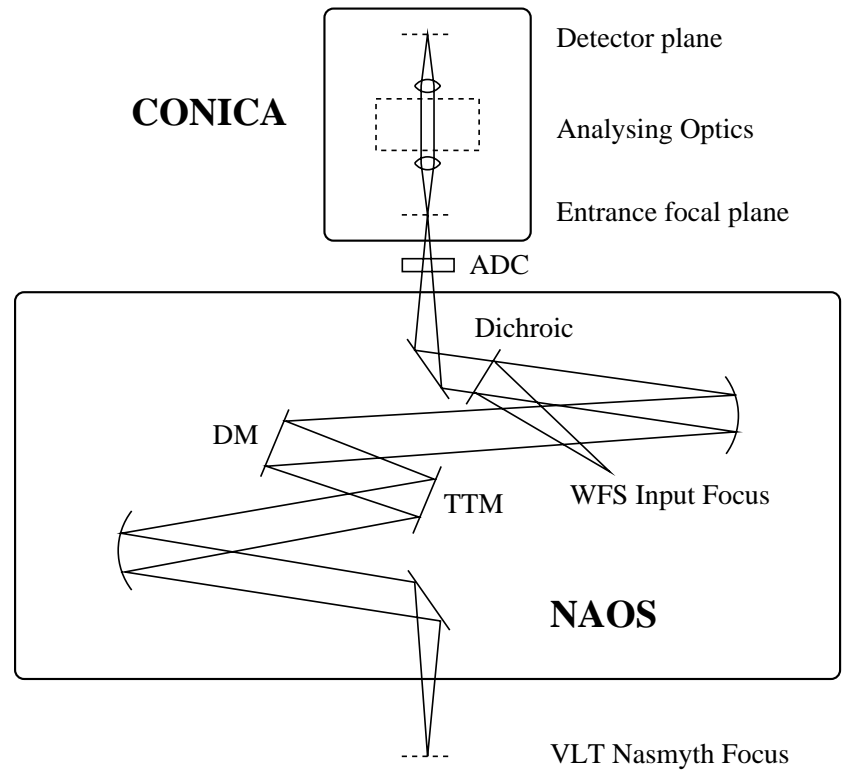

Fig. 1. Outline of the VLT instrument NAOS-CONICA.

Table 1. The required defocus distances for a phase diversity of $2 \pi$ rad (peak to valley) $(\lambda=1 \mu \mathrm{m})$ are listed corresponding to the f-ratios (pixel scales).

\begin{tabular}{lllll}
\hline \hline Camera & $f / D$ & Pixel scale & $d(\mathrm{~mm})$ \\
\hline $\mathrm{C} 50 \mathrm{~S}$ & 51 & 13.3 & $\mathrm{mas} /$ pixel & 20.8 \\
$\mathrm{C} 25 \mathrm{~S} / \mathrm{C} 25 \mathrm{~L}$ & 25.5 & 27.1 & $\mathrm{mas} /$ pixel & 5.2 \\
$\mathrm{C} 12 \mathrm{~S} / \mathrm{C} 12 \mathrm{~L}$ & 12.8 & 54.6 & $\mathrm{mas} /$ pixel & 1.3 \\
C06S/C06L & 6.38 & 109 & $\mathrm{mas} /$ pixel & 0.33 \\
\hline Entrance focal plane & 15 & 1.72 & $\mathrm{mas} / \mu \mathrm{m}$ & 1.8 \\
\hline
\end{tabular}

one with the long wavelengths region (L-camera). The only exception is the camera with the highest magnification (C50S). There is no long wavelength counterpart needed ${ }^{3}$.

A variety of different observing modes is provided by the analyzing optics: chronography, low resolution long slit spectroscopy, imaging spectroscopy by a tunable cold Fabry-Perot, polarimetry by wire-grids or Wollaston prisms, and about 40 broad- and narrow-band filters can be chosen.

\section{Phase diversity setup}

Our input for phase diversity wavefront estimation are two images: one of them in focus and the other one out of focus. In this manner we introduce the well-known phase diversity which is an obligatory input parameter for PD. One should recall that best phase diversity estimates are to be expected applying a peak to valley phase diversity $\Delta \phi$ between $1 \pi$ and $3 \pi$ and the input images must be at least Nyquist sampled ${ }^{4}$ (Paper I).

\footnotetext{
The Nyquist criterion for $L$ and $M$-band is already fulfilled for a camera with lower magnification $(\mathrm{C} 25 \mathrm{~L})$.

${ }^{4}$ Indeed, it is possible to model the wavefront error even at undersampled images (Jefferies et al. 2002). In our case there is no cost to be constrained by the Nyquist criterion because we can rather select the appropriate filter or else we deal with a camera used in the $L$ - and $M$-band where static wavefront aberrations are negligible.
} 


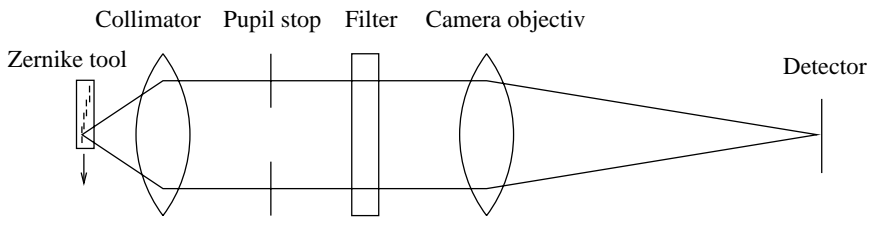

Fig. 2. The Zernike tool with its pinholes in the light path of CONICA.

The corresponding defocus distance $d$ depending on the applied wavelength is obtained by

$d=\frac{4 \lambda}{\pi}(f / D)^{2} \Delta \phi$

In the next two subsections we describe in detail two ways of introducing this phase diversity. Both ways are essential to enable us to separate the wavefront error and to assign it to different contributors. This disentanglement is described in Sect. 4.2.

\subsection{CONICA stand-alone: Focus shift by object}

First, we regard the possibilities to obtain the necessary input images with CONICA stand-alone. The CONICA detector is mounted on a tunable stage which is software controlled and can be driven in the cold environment. This allows us in principal to obtain a defocused image but the focus drive spans only a region of $2 \mathrm{~mm}$. Using Eq. (1) we compile for all available camera objectives the necessary defocus distances in the detector plane corresponding to a diversity of $2 \pi$ at a wavelength of $1 \mu \mathrm{m}$ in Table 1 . Only for the low magnification cameras (C06, $\mathrm{C} 12$ ) is the defocus distance sufficient. But these very cameras undersample in $K$ and at shorter wavelengths so that the focus stage mechanism finally fails in every case. For that reason we swerve to the entrance focal plane. Here a phase diversity of $2 \pi$ corresponds to $1.8 \mathrm{~mm}$ at a wavelength of $1 \mu \mathrm{m}$ or $3.6 \mathrm{~mm}$ at $2 \mu \mathrm{m}$, which is small enough to be implemented in the entrance focal plane.

In this plane a wheel is located carrying different field limiting masks, coronographic masks and the slits for spectroscopy. On the wheel we implement four different pinholes at different axial positions. The pinhole diameter is $10 \mu \mathrm{m}$. One pinhole is placed exactly in the entrance focal plane and yields a focused image on the detector $(0 \mathrm{~mm})$, and three other pinholes are located $1 \mathrm{~mm}, 2 \mathrm{~mm}$ and $4 \mathrm{~mm}$ out of the entrance focal plane. The four pinholes are mounted onto a plate fitting in a socket of the mask wheel. This device will be referred to as a Zernike tool later on. Impacts on the PD estimation due to the mechanical precision of the pinhole positions and possible deviations of their shape are investigated in Paper I.

Figure 2 depicts the setup for the CONICA internal phase diversity measurements. The leftmost component carries the four pinholes, which are shifted against each other, with the values given above. In rotating the wheel holding the Zernike tool we are able to select a pinhole in the field of view. After a collimating lens and a pupil stop, a filter selects the wavelength range and finally the camera objective forms the object image on the detector. The chosen camera objective determines the f-ratio and the pixel scale.
To center the image of the pinholes on the detector, the whole pinhole mount is shifted by turning the mask wheel. In principal PD needs the input images to be on the same spot to ensure that the same aberrations are sensed. The horizontal position of the pinholes can be controlled by adjusting the rotation angle of the wheel. In vertical direction there is no degree of freedom, but the four pinholes are mounted circularly to compensate for the circular movement. By this means a vertical precision of 50 mas (C50S) can be reached ${ }^{5}$. This is easily sufficient not to see any influence due to field aberration effects. PD measurements taken at different detector positions and calculations performed with an optical design software showed that even at the corner of the field of view $(13 \mathrm{arcsec})$ the field aberration is negligible (Paper I). Note that for some measurements in Paper I an earlier version of the Zernike tool was used with a design not optimized for the circular movement of the pinholes. The worst separation that could occur with the former Zernike tool was about 1.3 arcsec. But even with this tool no relevant impact on the precision of wavefront sensing was detected.

Apart from the fact that the Zernike tool with its pinholes at the entrance focal plane provides the required focus shifts, it is convenient that the required focus shifts do not depend on the camera objective (pixel scale) anymore. But note: defocusing by moving an object in the entrance focal plane does not correspond exactly to a defocus due to a shifted detector plane. An investigation of this effect is done in Paper I and turns out to be negligible.

To summarise this section: the PD input data to derive the total CONICA internal aberrations are obtained by object defocusing in the CONICA entrance focal plane. The object defocusing is realized by four $10 \mu \mathrm{m}$ pinholes at different axial positions. Note that since the entrance focal plane of CONICA is located inside the cold cryostat, aberrations accrued from the CONICA entrance window are not included in this wavefront estimation.

\subsection{NAOS-CONICA: Focus shift by the deformable mirror}

Now, we describe how the PD input images are obtained which are used to sense the wavefront aberrations of the whole instrument, i.e., the adaptive optics NAOS together with its infrared camera CONICA. In this case we can take advantage of the AO system's capabilities to itself introduce an adequate focus shift and thus there is no need for the implementation of a special tool or a modifaction of the design.

In the entrance focal plane of NAOS, which coincides with the VLT Nasmyth focal plane, a calibration point source can be slid in and imaged by CONICA. This point source is realized by the output of a fiber with a diameter of $10 \mu \mathrm{m}$ fixed on a movable stage. On the same stage a second source much larger in diameter $(400 \mu \mathrm{m})$ is mounted. It is only seen by the WFS and serves as a reference source to close the loop. This extended source is needed for technical reasons. In the case of no atmospheric turbulence the more extended source

\footnotetext{
51 pixel corresponds to 13.3 mas (C50S).
} 


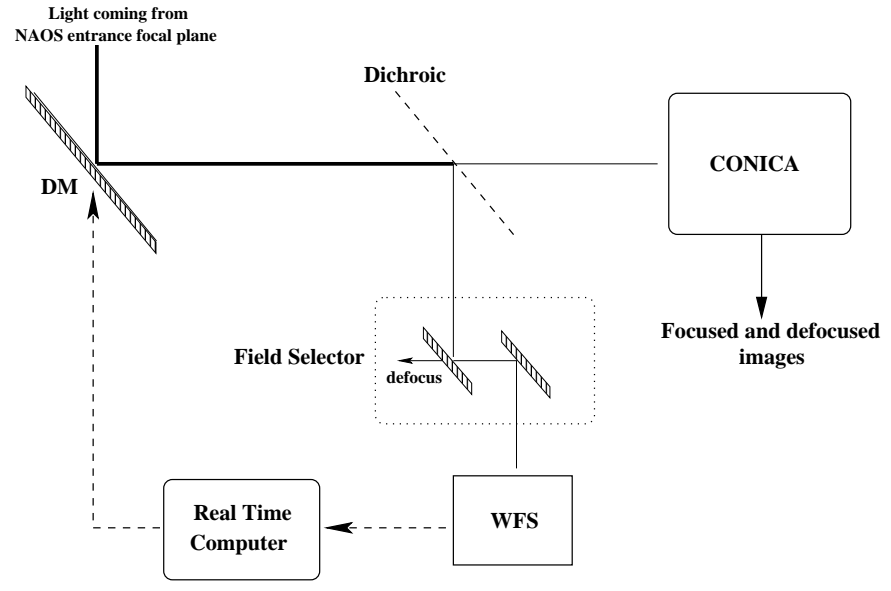

Fig. 3. Defocusing in closed loop using the NAOS Field Selector

provides a much better feed-back signal to the WFS than the small one. By this means the AO control loop is adjusted for any aberrations emerging in the common path. To obtain the focus shift affecting the entire instrument, we introduce the desired amount of defocusing in the WFS path by moving the mirrors of the field selector. During this process the loop is kept closed. Instantly, the arising focus shift is detected by the WFS. Correspondingly, the real time computer commands the DM to compensate for the detected defocus. Finally, the spots on the Shack-Hartmann WFS are centered again, but the defocus of the DM takes effect in the imaging path. For a pure defocus the DM will take a parabolic shape. The maximum achievable defocus by this method is limited by the DM's stroke and turns out to be about $20 \mathrm{~mm}$. Refering to Table 1, this is enough to introduce the needed diversity for an $\mathrm{f} / 15$ beam.

The procedure is shown in Fig. 3 and provides us with the PD input data to estimate the NAOS-CONICA overall wavefront errors. In comparison with the procedure described in Sect. 3.1 we deal with the same object now, and we must not care about any deviations in the position of the image pairs. This simplifies data aquisition for the measurement and diminishes the number of possible error sources.

\section{Calibration of NAOS and CONICA static aberrations}

\subsection{Introduction}

An appropriate way to describe the shape of a wavefront in the telecope pupil is by using Zernike polynomials (Noll 1976). A set of Zernike coefficients indicates the linear combination describing the present wavefront. As a matter of course, we can regard this set of coefficients as a vector. We refer to the Noll notation (Noll 1976) which labels the focus with 4 , the tangential and sagittal astigmatism with 5 and 6, coma with 7 and 8 , and so on. Since the coefficients for piston (1), and tiptilt $(2,3)$ are extraneous to the image quality they are dropped.

An extensive examination of the variety of error sources due to the practical and instrumental constraints was done in the preceding Paper I. The induced aberrations due to defocusing by a shifted object in the CONICA stand-alone case have been simulated and proven to be negligible. The influence of the pupil shape and its numerization have been evaluated, errors taken in account with regard to the camera pixel scale and the defocus distance deviations have been simulated and the problem of different object structure was considered. Furthermore we focused in detail on the handling of data reduction, e.g. the influence of the different noise sources such as readout noise or pickup noise. In Paper I we state that all these error sources accumulate to $\pm 35 \mathrm{~nm} \mathrm{rms}$ for the focus coefficient (4). Since the presented calibration data of this paper are acquired with an optimized Zernike tool, the expected error should be well below this number. The accuracy of the higher order coefficients has not changed and amounts to about $\pm 5 \mathrm{~nm} \mathrm{rms}$.

In this section we describe how the overall wavefront error can be decomposed and assigned to its corresponding optical components. Then we present the experimental results for one camera objective and some selected filters of CONICA as well as the results for the dichroics of NAOS.

\subsection{Disentanglement}

In the preceding sections we described how the static noncommon-path wavefront error can be measured, whether for CONICA stand-alone or for the entire instrument NAOSCONICA. However, each determination of the wavefront error is only valid for the particular instrument configuration in which it was measured. The tremendous number of instrument configurations ${ }^{6}$ makes it impractical to perform these calibrations for any possible instrument setup. For a practical application we need to split up the measured wavefront aberrations and assign the corresponding contributions to the divers optical components. This allows the construction of a configuration table $^{7}$ with entries for each optical component of the instrument. When a special instrument configuration is selected, the corresponding wavefront error contributions can be read out, added together and delivered to the AO system. This enables the DM to pre-correct for the current static wavefront aberrations.

In principal, we have to differentiate between three categories of optical components in the imaging path: the NAOS dichroics, the CONICA filters and the camera objectives.

The contribution of the NAOS dichroics $a_{i}^{\text {dichro }}$ can be determined by subtracting the overall NAOS-CONICA instrument aberrations $a_{i}^{\text {NCtot }}$ from the total CONICA instrument aberrations $a_{i}^{\text {Ctot: }}$

$a_{i}^{\mathrm{dichro}}=a_{i}^{\mathrm{NCtot}}-a_{i}^{\mathrm{Ctot}}$.

The vector components are labeled by the Zernike number $i$ running in our case from 4 to 15 . Regarding the PD estimations of the different CONICA filters for one camera objective (see Fig. 4 and Sect. 4.3) we ascertain that generally the filter aberrations $a_{i}^{\text {fil }}$ are small and mainly the achromatic camera

\footnotetext{
6 Given the combinations of 5 NAOS dichroics, 40 narrow and broad band filters and 7 camera objectives!

7 In fact, a configuration file is generated. The instrument control software takes care of what coefficients have to be applied for the selected instrument setup. These processes are hidden and completely automatic.
} 


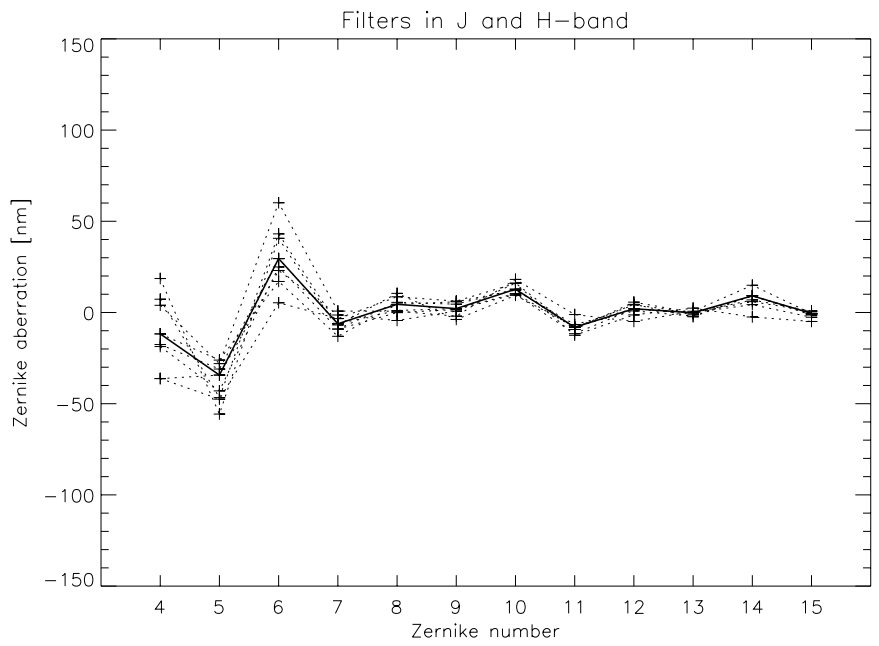

Fig. 4. CONICA internal aberrations measured by $8 \mathrm{NB}$ filters in $J$ and $H$-band with camera objective C50S and pinhole pair $0 / 2 \mathrm{~mm}$. The thick line indicates the median representing the camera aberrations.

objective aberrations are seen. In any case the filter aberrations are not correlated with each other nor with the camera ones. This suggests that we can deduce the camera objective contribution by applying the median to the total CONICA internal aberrations $a_{i}^{\text {Ctot,fili }}$. We prefer the median instead of the mean to avoid taking into account highly aberrant filters. The filter $i$ which was used to determine the corresponding total CONICA internal aberration $a_{i}^{\text {Ctot }}$ is indicated by fili. The camera objective and the residual filter contributions are obtained by these relations:

$a_{i}^{\mathrm{cam}}=\operatorname{median}\left(a_{i}^{\mathrm{Ctot}, \mathrm{fil} 1}, a_{i}^{\mathrm{Ctot}, \mathrm{fil} 2}, \ldots, a_{i}^{\mathrm{Ctot}, \mathrm{fil} n}\right)$

$a_{i}^{\mathrm{fil}}=a_{i}^{\mathrm{Ctot}}-a_{i}^{\mathrm{cam}}$.

The separation of the wavefront aberration into the contributions associated with the three categories of optical components (NAOS dichroics, CONICA filters and camera objectives) is only possible when we make use of both ways to introduce a focus shift, i.e. the DM to determine the NAOS-CONICA overall aberrations and the Zernike tool to determine the total CONICA aberrations. In addition, we note that even if we refer to these three categories by the notation dichroics, filters and objectives, the other components in the optical path are included, as well, even when they are not mentioned explicitly. E.g., the aberrations of the CONICA entrance window are included in the dichro aberrations and the aberrations of the CONICA collimator are an inextricable part of the camera aberrations.

\subsection{Calibration of CONICA: Camera and filters}

Figure 4 shows the aberrations for all eight narrow band filters in $J$ - and $H$-band of CONICA. The camera objective C50S and the pinhole pair $(0 / 2 \mathrm{~mm})$ is used to obtain the calibration data. The fourth coefficient $a_{4}^{\text {Ctot,fili }}$ expressing the defocus shows a

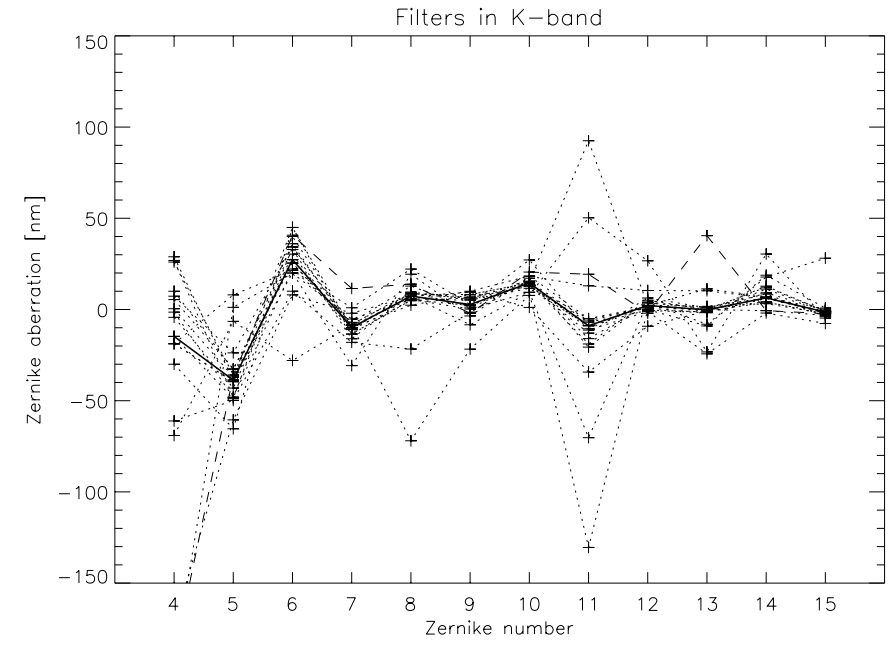

Fig. 5. CONICA internal aberrations measured by 19 NB filters in $K$-band with camera objective C50S and pinhole pair $0 / 4 \mathrm{~mm}$. The thick line indicates the median representing the camera aberrations. The dashed line highlights the aberrant filter NB2.09 which is picked out for the demonstration images in Fig. 6.

peak-to-peak variation of up to $60 \mathrm{~nm}$. This implies a slight imprecision of coplanarity of the filters in the cold environment. The other measured coefficients associated with the different filters noticeably resemble each other. This is evidence that these narrow-band filters contribute little to the total aberration of the system and mainly the camera objective aberration is seen.

Figure 5 displays the calibration results in the $K$-band. In total, 19 filters have been calibrated using the pinhole pair $0 / 4 \mathrm{~mm}$. One of the strongly aberrant filters (NB2.09) is highlighted by a dashed line. A large defocus in comparison to the other ones is detected. This filter is expected to have a striking error of coplanarity. It is not surprising that the strong defocus comes along with a particularly high spherical aberration $(i=11)$. The other highly aberrant filters show the same behaviour in comparison with the common filters of minor aberrations. The spherical aberration expresses the next order of a radial symmetric Zernike mode. The probability that a strong default of coplanarity induces only a defocus and does not concern higher orders is small. The PD input images of this aberrant filter is depicted at the bottom of Fig. 6. The right image shows the PSF registered in focus, and the left image a PSF having introduced a defocus of $4 \mathrm{~mm}$. Already the in-focus image reveals a strong degradation, but especially the phase inversion due to the high defocus can be clearly seen in the out of focus image. A bright spot emerges in the center of the "donut". On the top of this couple of images another couple of images is depicted. These are the PD input data of a filter (NB2.06) with normal behaviour and without strong aberrations.

As described in Sect. 4.2 the median of each Zernike number of the whole set of vectors yield the vector describing the camera contribution.

The accuracy of separating the camera aberrations from the raw aberrations (filters including camera) by the method described above is striking. The median aberrations for the filters of the two different wavelength regions plotted in Figs. 4 and 5 


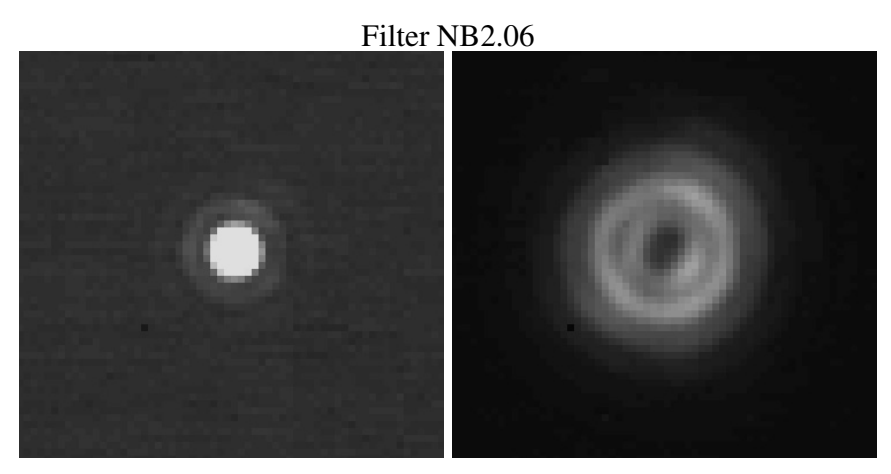

Filter NB2.09
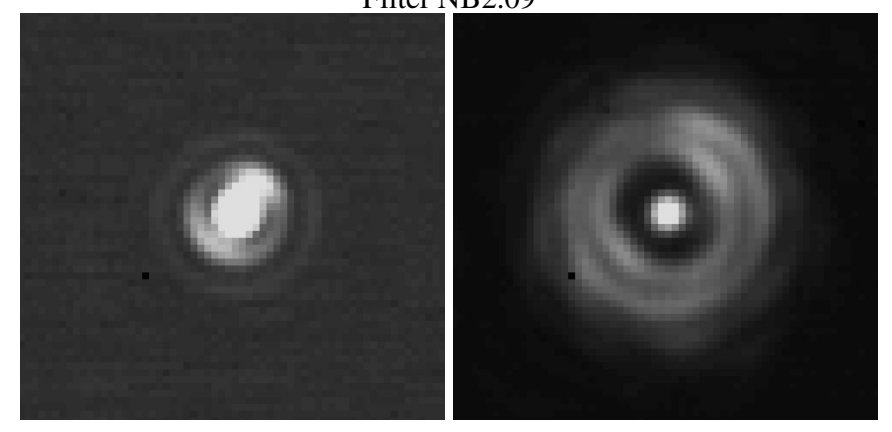

Fig. 6. Comparison of PD input images of a filter with small aberrations (NB2.06, on the top) and a filter with high aberrations (NB2.09 at the bottom). The in-focus images are placed on the left side, the out of focus images on the right side. The defocus distance is $4 \mathrm{~mm}$ for both filters (f/15).

are compiled in Table 2. The deviations of both median values are clearly below the expected error (see Sect. 4.1). Table 2 lists these median coefficients taken from all NB filters in $J$-, $H$ - and $K$-band. Keeping in mind that the achievable precision is a few nm we state that the camera aberrations are very small. The highest contributions arise from the focus term (4) and the astigmatism $(5,6)$. Section 5.3 gives an idea of the impact on the image quality dealing with Zernike mode aberrations in this order of magnitude. The residual filter aberrations are obtained by Eq. (4). In general, besides the focus coefficient and a few deviating filters these values are close to zero, too.

\subsection{Calibration of NAOS: Dichroics}

The calibration data are obtained with the fiber at the entrance focal plane of NAOS using the adaptive optic system itself for defocusing (see Sect. 3.2). Since the Zernike coefficients for the NAOS dichros are determined differentially, i.e. by subtraction of the total CONICA aberrations from the NAOS-CONICA overall aberrations, we can choose any reference camera and filter to perform the measurements as long as the components stay the same. A good choice is camera objective C50S and filter FeII1257. This objective oversamples even in the $J$-band and the filter has a small wavelength and therefore yields a higher accuracy in sensing wavefront errors. A suitable distance for the focus shift at this filter wavelength in the f/15beam is $2 \mathrm{~mm}$. We can calculate suitable defocus distances using Eq. (1).
In the following the properties of the five NAOS dichroics are itemized:

- VIS: Visible light to WFS; $J, H, K, L$ and $M$ to CONICA;

- N20C80: $20 \%$ of the incoming light to WFS; $80 \%$ to CONICA $(J, H, K)$;

- N90C80: $90 \%$ of the incoming light to WFS; $10 \%$ to CONICA $(J, H, K)$;

- $K: K$ to NAOS; $J$ and $H$ to CONICA;

- JHK: $J, H$ an $K$ to NAOS; $L$ and $M$ to CONICA.

Four of these five dichroics have been calibrated. The dichroic $J H K$ is omitted since only light in $L$ and $M$ band reaches CONICA. It is unreliable to sense the small wavefront errors of NAOS-CONICA at these wavelengths. Furthermore there is no need to, because the small static aberrations become completely negligible in $L$ and $M$.

The calibration results are compiled in Table 3 and Table 4. The first table lists the direct PD results. Any correction performed by these coefficients would only apply to the instrument configuration that was used to obtain the calibration data. The second table lists the aberrations directly assigned to the dichroics. These were obtained by subtracting the total CONICA aberrations that have been measured with the same filter and camera objective using the Zernike tool. It is noteworthy that the sensed astigmatism (Zernike number 5, 6 ) in the separated case is higher than in the overall case. Obviously a part of the camera astigmatism is compensated by the dichroics.

It is noteworthy that this tendency applies for all dichroics. Different reasons can cause this behaviour. First, the inclination of the dichroics artificially introduce an astigmatism. Even if the NAOS dichroics are designed for prism shape and do correct for this effect, a residual error cannot be excluded. Furthermore a certain amount of astigmatism can be introduced by components other than the dichroics lying in the same part of the light path, e.g. the output folding mirror or the CONICA entrance window (see Fig. 1). Nevertheless, it is not a limitation of the calibration method but only a question of assigning the contribution of the wavefront errors to the different optical components. In the end, only the sum of all aberrations has to be correct.

\section{Image quality versus estimated aberrations}

\subsection{Strehl ratios by $P D$ and focal plane image}

The PD calibration data can be used to investigate the available image quality in different ways. First, the knowledge of the wavefront allows us to calculate a SR. After the reduction of the calibration data the wavefront is described by a set of Zernike coefficients. Furthermore, we can just refer to the infocus image and calculate a SR with the measured point spread function. In the following we give a more detailed explanation of how these SRs are obtained. 
Table 2. Camera aberrations in nm RMS by the median over the filter + camera aberrations in the bands $J, H$ (pinholes $0 / 2 \mathrm{~mm}$ ), $K$ (pinholes $0 / 4 \mathrm{~mm})$ and all bands $(J, H, K)$.

\begin{tabular}{crrrrrrrrrrrr}
\hline \hline Bands for median & 4 & 5 & 6 & 7 & 8 & 9 & 10 & 11 & 12 & 13 & 14 & 15 \\
\hline$J, H$ & -12 & -34 & 30 & -6 & 4 & 2 & 13 & -8 & 2 & 0 & 9 & -1 \\
$K$ & -15 & -39 & 27 & -10 & 7 & 3 & 15 & -9 & 2 & 0 & 6 & -1 \\
$J, H, K$ & -15 & -39 & 27 & -9 & 6 & 2 & 13 & -9 & 2 & 0 & 7 & -1 \\
\hline
\end{tabular}

Table 3. NAOS dichros, overall NAOS-CONICA aberrations in nm rms, reference filter: FeII1257.

\begin{tabular}{lrrrrrrrrrrrr}
\hline \hline Dichro & 4 & 5 & 6 & 7 & 8 & 9 & 10 & 11 & 12 & 13 & 14 & 15 \\
\hline VIS & 15 & -5 & 24 & -6 & 23 & 5 & -8 & -9 & 7 & -13 & -7 & 3 \\
N20C80 & 2 & -1 & 42 & -2 & 30 & 5 & -4 & 14 & -1 & -19 & -8 & 4 \\
N90C10 & -7 & -3 & 36 & -3 & 19 & 6 & -5 & -28 & 1 & -9 & -9 & 1 \\
K & -8 & 14 & -17 & -4 & 18 & 3 & -5 & -6 & 7 & -14 & -10 & 2 \\
\hline
\end{tabular}

Table 4. Separate NAOS dichro aberrations in nm rms.

\begin{tabular}{lrrrrrrrrrrrr}
\hline \hline Dichro & 4 & 5 & 6 & 7 & 8 & 9 & 10 & 11 & 12 & 13 & 14 & 15 \\
\hline VIS & -18 & 37 & -5 & 3 & 16 & 2 & -21 & -44 & 6 & -13 & -14 & 6 \\
N20C80 & -32 & 41 & 13 & 8 & 23 & 1 & -17 & -21 & -3 & -18 & -15 & 6 \\
N90C10 & -41 & 38 & 7 & 7 & 12 & 3 & -18 & -64 & -1 & -9 & -16 & 4 \\
K & -42 & 56 & -47 & 5 & 11 & -1 & -18 & -42 & 5 & -14 & -17 & 5 \\
\hline
\end{tabular}

\section{Strehl by PD}

For small wavefront deviations the SR can be determined via the coherent energy referring to the wavefront variance $\sigma^{2}$ in radian. The PD estimation yield the wavefront expanded in terms of Zernike coefficients $a_{i}$. For small $\sigma^{2}$

$\mathrm{SR} \simeq \mathrm{e}^{-\sigma^{2}} \simeq 1-\sigma^{2} \simeq 1-\sum_{i=4}^{m} a_{i}^{2}$

allows us to calculate the SR directly by the output of PD estimation. In principal the sum runs to infinity $(m=\infty)$ but for our purpuse we stop at $m=15$. We can compare these SR numbers to the ones that are directly determined by the in-focus images.

\section{Strehl on image}

A straight-forward way to calculate a SR on the focus image (PSF) is to construct a theoretical diffraction-limited image $\mathrm{PSF}_{\text {diff }}$ taking into account the wavelength, the pixel scale, the aperture and the central obscuration ${ }^{8}$. Having normalized the total intensity of the PSF and PSF $_{\text {diff }}$ to 1, the fraction of these values yields the SR (see Eq. (6)).

In particular, in the case of the PSF sampling being close to the Nyquist criterion this approach has the disadvantage of being sensitive to the exact position of the PSF peak with respect to the pixel center. Furthermore, since the total intensity has to be determined by the integrated signal over a wider region around the PSF, the reliability of the SR value depends on a precise background correction. If the background is overestimated, then the SR will be overestimated, too, and vice versa.

8 The central obscuration which is caused by the secondary mirror will decrease slightly the central peak intensity and raise the side lobes of the Airy function.
The reliability of the SR values can be enhanced when we switch from the image space to the Fourier space by

$\operatorname{SR}=\frac{\operatorname{PSF}(\alpha=0)}{\operatorname{PSF}_{\text {diff }}(\alpha=0)}=\frac{\int \operatorname{OTF}(f)}{\int \operatorname{OTF}_{\text {diff }}(f)}$,

where OTF is the optical transfer function. Since in Fourier space only spatial frequencies are considered, a shift of the PSF is of no importance anymore. Aside from that, an elegant and reliable background correction can be performed using the zero spatial frequency. We calculate the SRs by the following procedure $^{9}$ :

- The image is corrected by its corresponding background;

- The OTF is calculated. It is given by the real part of the Fourier transform of the image;

- The residual background is corrected by the zero frequency. A fit of the very first spatial frequencies is used to extrapolate the true zero frequency value. The difference of the measured and the extrapolated value for the zero frequency yields the residual background;

- The noise level is subtracted using the high frequencies beyond the diffraction limit;

- A theoretical telescope OTF is constructed and multiplied by a Bessel function to account for the spatial spread due to the object size;

- The detector response is taken into account. This is done by a further multiplication of the theoretical telescope OTF with the Fourier transform of the detector response;

- The SR is obtained by the division of the normalized integrals from the measured and the theoretical OTF. All points with spatial frequencies higher than the diffraction limit are excluded.

\footnotetext{
9 This routine was developed by T. Fusco, ONERA.
} 


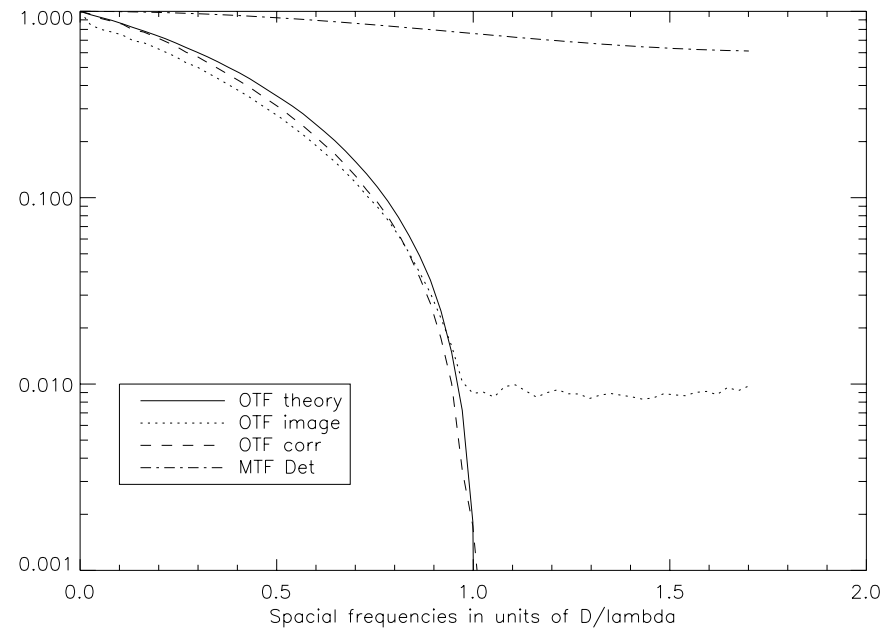

Fig. 7. Visualization of the theoretical and measured OTF at the example of filter FeII1644.

Figure 7 gives an example of how the described OTFs look for a PSF taken through the filter H2(1_0)S(7). To allow for a one dimensional representation, the circular mean of the two dimensional OTF is calculated. The raw, untreated OTF of the image is labeled "OTF image". "OTF corr" displays the image OTF which is corrected for the residual background at zero frequency and for the noise level. The noise level of the uncorrected image OTF can be seen as plateau beyond the cutoff frequency $D / \lambda$ and averages $1 \%$ of the maximum value. The theoretical diffraction-limited telescope OTF includes the reduction due to the object size ${ }^{10}$ and the correction for the detector modulation transfer function (MTF). It is labeled "MTF Det" and is located at the top of the plot. The detector response is constructed by the assumption that roughly $3 \%$ of the total intensity is contained at each of the adjacent pixels and $1.5 \%$ in the corner pixels. Here we refer to Finger et al. (2000). In this paper a measurement of the response of a comparable infrared array is described. Due to the lack of a precise knowledge of the detector response it is constructed by a quadratic scaling in relation to the different pixel size ${ }^{11}$. A quadratic scaling is implied by a linear behaviour of the diffusion of the minority carriers in the detector material in one dimension for small distance variations.

\subsection{Comparison of Strehl ratios}

The resulting SRs for the narrow-band filters in $J, H$, and $K$ are presented in Figs. 8 and 9. For each filter two SRs are given: the SR by PD and the SR on the image.

A number of error sources contribute to the error of the SR values on image. Beside of small error contributions due to uncertainties of the pixel scale and the flatfield, the remaining uncertainty of the background correction and the detector

\footnotetext{
${ }^{10}$ Since a $10 \mu \mathrm{m}$ pinhole is used which is barely resolved, the influence is small and amounts for the smallest wavelength $(1 \mu \mathrm{m})$ at most to $5 \%$.

${ }^{11}$ The pixel size of the CONICA detector (Aladdin $1 \mathrm{~K} \times 1 \mathrm{~K}$ ) is $27.0 \mu \mathrm{m}$.
}

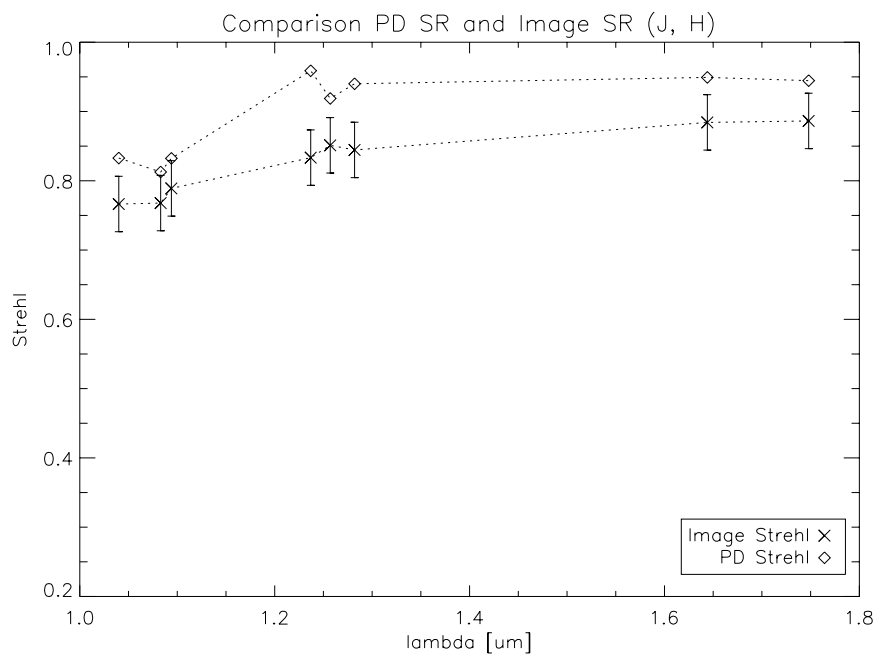

Fig. 8. Comparison of SR versus wavelength in $J$ and $H$ band calculated directly and derived from PD results.

response lead us to estimate an absolute error of $\pm 4 \%$. The expected wavelength dependency of the MTF error is minor with respect to the remaining background error. Therefore it is neglected and we use the constant value given above derived from experience in reducing the experimental data.

Recall that the SR by PD has a maximal wavelengthdependent error of $\pm 5 \%$ at $1 \mu \mathrm{m}$ and $\pm 1 \%$ at $2 \mu \mathrm{m}$ taking into account an error of $\pm 35 \mathrm{~nm}$ RMS for the focus estimation $(i=4)$. The main contributor to this error is a systematic error in the precision of the pinhole positions in the Zernike tool (see Sect. 4.1).

In general the PD SRs exceed the other SR values. This reflects the fact that the wavefront is expanded by a limited number of Zernike coefficients and the higher order aberrations are cut off. Note that it is not astonishing that in the case of very low SR values (worse than 50\%) the PD SR value may lie below the image SRs (Fig. 9). Such strong wavefront errors violate the condition under which Eq. (5) is valid. Thus, we expect Eq. (5) to yield underestimated values.

The comparison of the SR values determined by the different methods turns out to be consistent. The longer the wavelength, the more the image and PD SR values approach each other. This shows that the influence of aberrations scales with the wavelength. In other words, the fact that we cut off at a certain Zernike number $(i=15)$ has a greater impact at short wavelengths.

\subsection{Focus adjustment}

Having in mind the small estimated wavefront errors that we presented in the previous sections we become conscious of the required precision of the most trivial aberration we regard: the focus. It is striking that even in the focus determination we depend on the precision of PD calibration. This becomes evident when we look at the conventional procedure of focus tuning and regard the loss of SR caused by the detected aberrations.

To tune the focus of CONICA, the in-focus pinhole of the Zernike tool is imaged on the detector. Now, a focus curve 


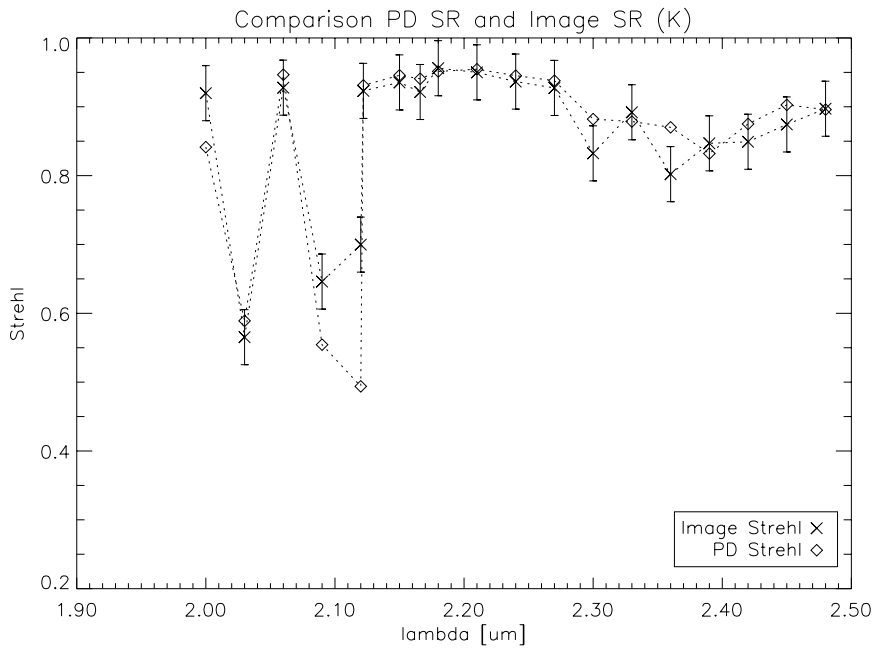

Fig. 9. Comparison of SR versus wavelength in $K$ band calculated directly and derived from PD results.

is obtained by taking images at different axial position of the detector stage (see Sect. 3.1). The maximum of the SRs indicate the proper focus position of the stage. The maximum of the obtained focus curves for the different cameras can be located with an accuracy of about $50 \mathrm{~nm} \mathrm{rms}$. For this wavefront error, Eq. (5) yields a loss of SR of $2.5 \%$ at a wavelength of $2 \mu \mathrm{m}$ and almost $10 \%$ at $1 \mu \mathrm{m}$. Thus, in particular in the $J$ - and $H$-band, the inaccuracy of determining the focus only by moving the detector stage gives reason for a significant loss of SR. Furthermore the whole effort of fine-tuning for the remaining static wavefront aberrations becomes irrelevant when the remaining focus error is in the regime of the highest higher-level aberrations (Zernike number $i \geq 5$ ). Compare the aberrations for focus with astigmatism in Figs. 4 and 5. The only way to achieve a significant improvement of the wavefront error, and therefore of the SR after closed loop compensation, is to ensure that the residual focus deviation is corrected properly, too. This is guaranteed by following the procedure:

- Determination of the rough nominal focus position of the CONICA detector for each camera with one reference filter. The in-focus pinhole of the Zernike tool serves as a reference;

- Determination of the nominal focus for the whole instrument. The calibration point source in the NAOS entrance focal plane serves as a reference. The data points for the focus curve are obtained by moving the field selector in closed loop. This has to be done for every NAOS dichroic;

- The corresponding data base entries are updated by the nominal focus positions (CONICA internal and NAOS). The nominal focus deviations are included in the data base. They are compensated for by moving the field selector in the case of switching the NAOS dichroics and by moving the CONICA detector stage in the case of switching the camera objectives;

- Then the PD estimation reveals the residual focus error for each configuration, in particular for each filter. They are entered into the data base together with the higher order aberrations. For a certain instrument configuration the corresponding values are fetched automatically and delivered to the AO system. The DM corrects for the residual focus deviations.

\section{Closed loop compensation of NAOS-CONICA static aberrations}

\subsection{Rendering of aberrations}

Having explored in detail the application of phase diversity to calibrate NAOS and CONICA static aberrations in Paper I, we presented above the experimental results applying PD as a wavefront sensor. We described how the contributions of the different optical components in the light path are separated to create a complete calibration configuration table. For each possible configuration of the instrument the corresponding correction coefficients are rendered to NAOS and are used to adjust the AO system. In this manner the DM will take the shape needed for compensation of the static wavefront aberrations. To demonstrate the final gain in optical quality we compare the originally acquired images without correction for static aberrations with the images obtained after closed loop compensation. The gain will be quantified in terms of SR numbers.

\subsection{Full AO correction}

The $10 \mu \mathrm{m}$ calibration source in the entrance focal plane of NAOS simulates a star without turbulence. The visible WFS is used to correct for the common path aberrations. Therefore, the loop is closed on the $400 \mu \mathrm{m}$ source as described in Sect. 3.2. The light is separated by the dichroic VIS, thus the WFS sees the visible part and the near-infrared is directed towards CONICA.

Figure 10 shows two extreme cases of applying AO compensation. The upper pictures demonstrate the correction for a filter in $J$-band, the pictures below in $K$-band. In accordance with Figs. 4 and 5 the sensed aberrations in $J$ and $K$ band are very similar - recall that the main contribution arises from the achromatic camera objective and the NAOS dichroic. But even if similar correction coefficients are rendered to the AO system, the effect on the image is strongly wavelength dependent. This is due to the fact that the influence of the applied Zernike coefficients scales with the wavelength. Thus, we achieve a striking correction in $J$-band visible with the naked eye on the images before and after correction. The most important aberration, the astigmatism, vanishes and the PSF is contracted. In $K$-band the non-corrected image is already very close to the optimum and the improvement is hard to see directly on the image. But calculating the SRs shows that even in $K$-band the performed correction is still significant (Table 5). Note that the given error arises from a maximum estimate of all error sources as described in Sect. 5.2. The nature of the error is mainly systematic (e.g., caused by background correction) and affects the calculated SRs for the image pairs in the same way. SRs determined on experimental data are intrinsically afflicted by rather high error bars, but a direct inspection of the images (central intensity, shape of the diffraction rings) shows the relative gain of 2 to $3 \%$ in $K$-band to be true. Even this rather small appearing gain in 


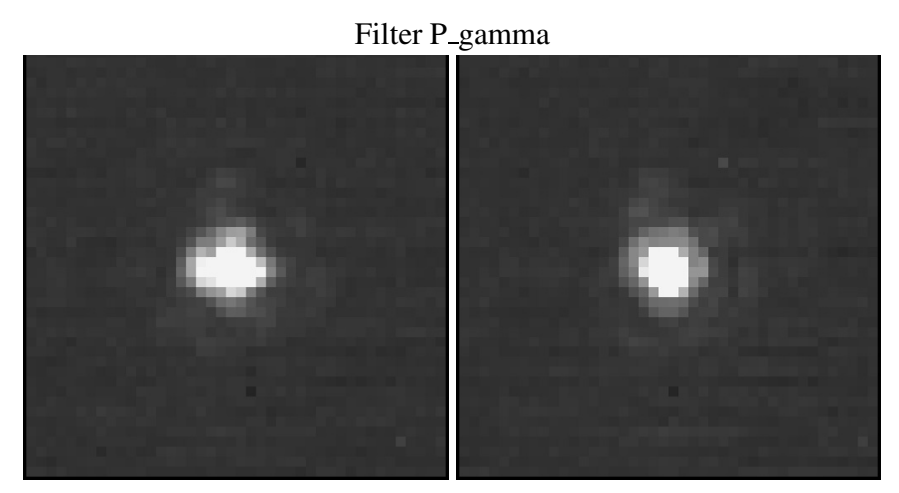

Filter Ks

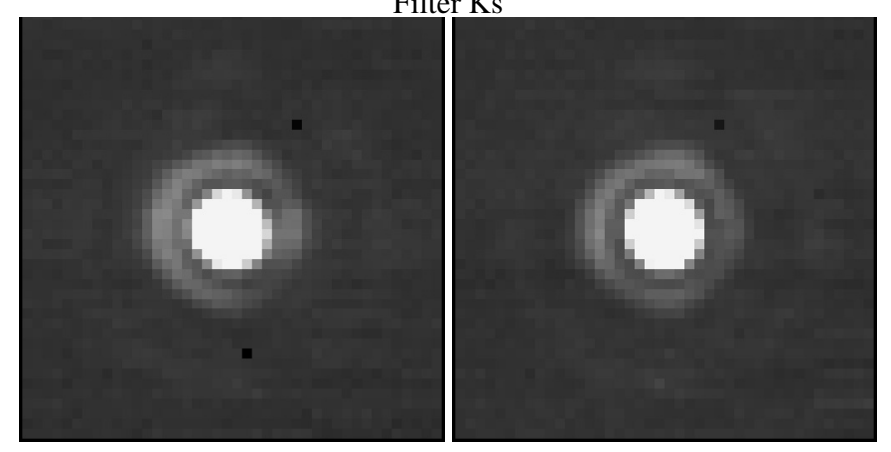

Fig. 10. Comparison of PSFs before and after closed loop compensation. Above a couple of $J$-band images and at the bottom a couple of $K$-band images are shown. Left side: without AO correction. Right side: with AO correction. Especially in $J$-band, the sharpening of the PSF can be clearly seen.

Table 5. Comparison of SRs for two selected filters before and after AO compensation for static aberrations. The maximum mainly systematic error is given. Statistical errors are significantly smaller.

\begin{tabular}{lcc}
\hline \hline Filter & SR no corr $(\%)$ & SR with corr $(\%)$ \\
\hline Pgamma & $60 \pm 4$ & $70 \pm 4$ \\
$K \mathrm{~s}$ & $91 \pm 4$ & $93 \pm 4$ \\
\hline
\end{tabular}

$K$ is of high importance. On the way to scientific goals such as e.g. planet detection, the total error budget must be tackled to eliminate every percentage point of loss in SR.

\section{Conclusion}

In Paper I we presented a guideline for a PD approach to calibrate static wavefront aberrations. An extensive investigation of system limitations and error sources has been carried out. This approach was shown to be very flexible and powerful for precise wavefront sensing using experimental data of the first VLT AO system NAOS-CONICA. In Paper II we have given a detailed description of its implementation at the instrument and presented the experimental results of the calibration data for a variety of observing configurations. Especially, we turned our attention to the disentanglement of the measured overall wavefront errors which allows a convenient allocation to the divers optical components and makes the calibration procedure feasible for an instrument with a huge number of possible configurations. The sensed wavefront errors expanded in Zernike coefficients have been used to quantify the image quality in terms of SR and be proven to be consistent with the SRs directly determined with the image data. Finally we gave a striking example of the acquired improvement of optical performance in comparing images with and without $\mathrm{AO}$ correction for static wavefront errors. It turned out that even starting with a very good image quality, we still could achieve a significant gain in terms of the SR ratio.

The design of the instrument control software is harmonized with this calibration procedure and the AO loop parameters are automatically updated when the instrument setup changes. This ensures that the utmost optical performance is provided for all the configurations. Additionally, this implementation of wavefront sensing can be used to monitor the optical quality and to alert for small degradations of optical performance. In general, for future high performance AO systems, the presented technique is of great importance to achieve the challenging science goals of the astronomical community. It should be included in the instrumental design at a very early phase.

Acknowledgements. We would like to thank Eric Gendron and Wolfgang Brandner for their patience and assistance in the fine tuning of the instrument and interpretation of the measurements during the commissioning runs. Furthermore, we thank Gert Finger for the fruitful discussions about the detector characteristics. We are thankful to Norbert Hubin for the assistance to this work on the part of the European Southern Observatory. This research was partially supported through a European Southern Observatory contract and the Marie Curie Fellowship Association of the European Community.

\section{References}

Blanc, A., Fusco, T., Hartung, M., Mugnier, L., \& Rousset, G. 2003, A\&A, 399, 373 ( Paper I)

Brandner, W., Rousset, G., Lenzen, R., et. al. 2002, The Messenger, 107,1

Finger, G., Mehrgan, H., Meyer, M., et al. 1998, in Infrared Astronomical Instrumentation, ed. A. Fowler, SPIE, 3354, 87

Gonsalves, R. A. 1982, Opt. Eng., 21-5, 829

Hartung, M., Bizenberger, P., Boehm, A., et al. 2000, in Optical and IR Telescope Instrumentation and Detectors, ed. M. Iye, \& A. Moorwood, SPIE, 4008, 830

Jefferies, S. M., Lloyd-Hart, M., Keith Hege, E., \& Georges, J. 2002, App. Opt., 41, 2095

Lenzen, R., Hofmann, R., Bizenberger, P., \& Tusche, A. 1998, in Infrared Astronomical Instrumentation, ed. A. Fowler, SPIE, 3354, 606

Noll, R. J. 1976, J. Opt. Soc. Am., 66-3, 207

Paxman, R. G., Schulz, T. J., \& Fienup, J. R. 1992, J. Opt. Soc. Am. A, 9,1072

Rousset, G., Lacombe, F., Puget, P., et al. 1998, in Adaptive Optical System Technologies, ed. D. Bonaccini, \& R. K. Tyson, SPIE, 3353, 508

Rousset, G., Lacombe, F., Puget, P., et al. 2000, in Adaptive Optical Systems Technology, ed. P. L. Wizinowich, SPIE, 4007, 72

Spanoudakis, P., Zago, L., Chételat, O., Gentsch, R., \& Mato Mira, F. 2000, in Adaptive Optical Systems Technology, ed. P. L. Wizinowich, SPIE, 4007, 408 